\title{
Inclusions in the Mechanical Capped Steel ${ }^{*}$
}

\author{
By Mamoru TOYOTA,** Katsukiyo MARUKAWA, ** Norihiko NOZAKI** \\ and Tsuguharu UEDA**
}

\section{Synopsis}

A study on the macroscopic inclusion in the rim and core zones of large mechanical capped steel ingots $(23 \sim 34 t \cdot$ weight $)$ has been made by using the slime method for extracting the inclusions.

(1) In the lenticular blow holes of the rim zone, there are many large macroscopic inclusions. A part of these inclusions is a glassy oxide of the (Fe, $\mathrm{Mn})-\mathrm{Si}-\mathrm{Al}-\mathrm{O}$ system, and the other part is a large lumpy inclusion which is composed of $\mathrm{Al}_{2} \mathrm{O}_{3}$.

(2) The former inclusions (glassy oxides) of a tortoise shell-shape stick on the inner side of lenticular blow holes, and the latter $\left(\mathrm{Al}_{2} \mathrm{O}_{3}\right)$, of a lumpy type, pops out into lenticular blow holes.

(3) In the blow holes of the core zone, there are large lumpy alumina inclusions, and there are more in the upper part of the core zone than in the middle and the bottom parts.

(4) These large lumpy inclusions are caught by the blow holes in the core zone and carried by them into ihe inner part of the zone.

(5) However, in the blow holes at the core zone, no inclusion of the tortoise shell-shape is found.

\section{Introduction}

Although numerous investigations have so far been made with respect to the inclusions in the rimmed steel and the findings have been reported in quantities, only few reports refer to the inclusions in the rim zone - in particular, macroscopic inclusions have been

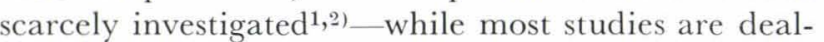
ing with the inclusions in the core zone.

The authors, meanwhile, recognized the existence of a large number of macroscopic nonmetallic inclusions in the rim zone of mechanical capped steel, and also discovered that a majority of such inclusions is captured within lenticular blow holes.
The inclusions captured by blow holes are classified into two sorts - the one is a low melting-point oxide of $\mathrm{Fe}-\mathrm{Mn}-\mathrm{Al}-\mathrm{Si}-\mathrm{O}$ system and the other is a large, lumpy, single-substance alumina.

The former is named tortoise shell-shape inclusion after its appearance, and the latter is termed lumpy alumina.

In addition, a number of large, lumpy alumina inclusions captured by blow holes was found in the core.

In this paper, it is mainly described the existing state of these macroscopic inclusions in the rim zone and the findings of investigation on lumpy alumina in the core. Throughout the present examination, the inclusions were extracted by the slime method.

\section{Method of Investigation}

The steel ingots examined are low-carbon, lowmanganese rimmed steel and medium-carbon, highmanganese rimmed steel, melted in a $250 \mathrm{t}$ converter at Kashima Works of Sumitomo Metal Industries, Ltd., and poured into a large-sized, bottle top mold.

The ingot making conditions, mold dimensions and other data on the steel ingots prepared for the investigation are roughly given in Table 1 .

After cooling down these steel ingots, ingot $\mathrm{A}$ was split along the center, parallel to the shorter side, to be used as a sample.

The macroetching photograph of ingot $\mathrm{A}$ is given in Photo. 1, in which black dots indicate the positions wherein the samples for the slime method were taken out.

Table 1. The condition of ingot making

\begin{tabular}{|c|c|c|c|c|c|c|c|c|c|c|c|c|c|}
\hline \multicolumn{2}{|l|}{ Ingot No. } & A & B & $\mathrm{C}$ & $\mathrm{D}$ & $\mathrm{E}$ & $\mathrm{F}$ & G & $\mathrm{H}$ & I & $\mathrm{J}$ & $\mathrm{K}$ & $\mathrm{L}$ \\
\hline \multirow{5}{*}{ Ladle analysis $(\%)$} & $\mathrm{C}$ & 0.06 & \multicolumn{2}{|c|}{0.07} & \multicolumn{2}{|c|}{0.07} & 0.07 & 0.06 & 0.07 & \multicolumn{2}{|c|}{0.18} & \multicolumn{2}{|c|}{0.16} \\
\hline & $\mathrm{Si}$ & 0.01 & \multicolumn{2}{|c|}{0.01} & \multicolumn{2}{|c|}{0.01} & 0.01 & 0.01 & 0.01 & \multicolumn{2}{|c|}{0.01} & \multicolumn{2}{|c|}{0.01} \\
\hline & $\mathrm{Mn}$ & 0.22 & \multicolumn{2}{|c|}{0.28} & \multicolumn{2}{|c|}{0.30} & 0.28 & 0.25 & 0.27 & \multicolumn{2}{|c|}{0.79} & \multicolumn{2}{|c|}{0.70} \\
\hline & $\mathrm{P}$ & 0.010 & \multicolumn{2}{|c|}{0.009} & \multicolumn{2}{|c|}{0.010} & 0.010 & 0.012 & 0.009 & \multicolumn{2}{|c|}{0.014} & \multicolumn{2}{|c|}{0.011} \\
\hline & $\mathrm{S}$ & 0.018 & \multicolumn{2}{|c|}{0.010} & \multicolumn{2}{|c|}{0.012} & 0.010 & 0.014 & 0.011 & \multicolumn{2}{|c|}{0.010} & \multicolumn{2}{|c|}{0.010} \\
\hline \multirow{3}{*}{\multicolumn{2}{|c|}{$\begin{array}{l}\text { Casting time }(\mathrm{min}) \\
\text { Capping time }(\mathrm{min}) \\
\mathrm{Al} \text { added in mold }(\mathrm{g} / \mathrm{t})\end{array}$}} & 3.7 & 3.0 & 2.7 & 2.2 & 2.2 & 3.7 & 3.3 & 3.8 & 3.4 & 3.9 & 3.0 & 3.5 \\
\hline & & 6.6 & 6.8 & 5.0 & 5.1 & 6.2 & 7.6 & 10.7 & 9.5 & 9.8 & 7.5 & 5.4 & 2.3 \\
\hline & & 30 & 30 & 9 & 36 & 15 & 15 & 30 & 36 & 2 & 9 & 6 & 0 \\
\hline \multicolumn{2}{|l|}{ Ingot weight (t) } & \multicolumn{8}{|c|}{33.6} & \multicolumn{4}{|c|}{23.0} \\
\hline \multirow{3}{*}{$\begin{array}{l}\text { Mold dimention } \\
\quad(\mathrm{mm})\end{array}$} & Width & & \multirow{3}{*}{\multicolumn{4}{|c|}{$\begin{array}{l}1725 \\
1040 \\
2950\end{array}$}} & & & & \multirow{3}{*}{\multicolumn{4}{|c|}{$\begin{array}{r}1431 \\
889 \\
2950\end{array}$}} \\
\hline & Thickness & & & & & & & & & & & & \\
\hline & Height & & & & & & & & & & & & \\
\hline
\end{tabular}

* Originally published in Tetsu-to-Hagané, 62 (1976), 362, in Japanese. English version received July $13,1976$.

** Kashima Works, Sumitomo Metal Industries, Ltd., Kashima-cho, Kashima-gun, Ibaraki 314. 
As for the remaining eleven ingots from $\mathrm{B}$ to $\mathrm{L}$, each corner was cut off to prepare a so-called corner sample. The samples for the slime-method were taken out of the rim zone at the middle $(50 \%)$ and bottom $(80 \%)$ parts of the ingot.

Again, the samples for the microscopic examination were chipped off from the $80 \%$ deep position of the ingot to measure the cleanliness of the inclusions according to the specified JIS method.

\section{Slime Method}

Several reports have been published ${ }^{1,3,4)}$ as to the slime method, and its practice is well known. However, the method suffers such disadvantages as the unsuitableness for mass treatment, and the formation of a large amount of electrolytic traces which will require several processes to separate the inclusions, possibly losing some inclusions in the course of these processes. To overcome such disadvantages, the authors devised the following means.

(1) A tray was placed under the sample during electrolysis so as to minimize the amount of traces containing inclusions to be extracted. For the application, it was confirmed that the inclusions would not drop off from the tray. Consequently, the quantity of traces was reduced to a considerable extent.

(2) The separation of the inclusions from the traces was greatly facilitated by reducing the amount of traces; the inclusions were almost completely separated by hydraulic elutriation with provisional use of a bar magnet. As a result of this treatment, both the treating amount and the precision were largely improved. In this experiment, a sample of nearly $3 \mathrm{~kg}$ was electrolyzed for about two weeks, in an electrolytic solution of $15 \%$ ferrous hydroxide solution, with 10 to $12 \mathrm{~V}$, and 8 to $10 \mathrm{~A}$.

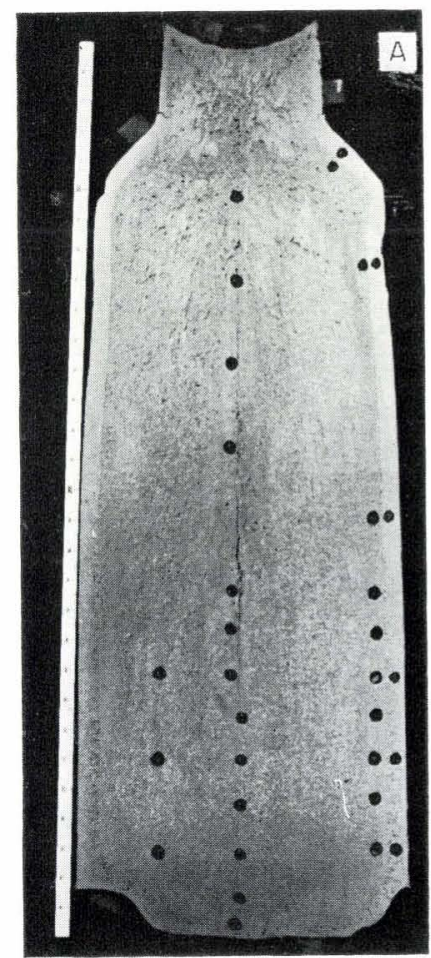

Photo. 1. Sampling positions for slime-method

\section{Findings}

\section{Inclusions in the Rim Zone}

\section{Extraction of Macroscopic Inclusions}

The quantitative results of the inclusions extracted from the rim zone by the slime method are given in Fig. 1.

From this examination, it was found that macroscopic inclusions of $50 \mathrm{mg} / 10 \mathrm{~kg}$ to $300 \mathrm{mg} / 10 \mathrm{~kg}$ and $20 \mathrm{mg} / 10 \mathrm{~kg}$ to $180 \mathrm{mg} / 10 \mathrm{~kg}$ are existing at the bottom and at the middle part of the ingot, respectively. Besides, it was also observed that a large portion of macroscopic inclusions has the size of more than $250 \mu$ and that there is no difference in the quantities of inclusions extracted from low-carbon rimmed steel (ingots $\mathrm{B}$ through $\mathrm{H}$ ) and medium-carbon rimmed steel (ingots I through L).

\section{Shape of Inclusions}

Some of the typical appearances of macroscopic inclusions extracted from the rim zone are represented in Photo. 2. The principal feature of these inclusions is that the semicircular (left), plane (middle) and bowllike (right) shapes are predominant in number as compared with the spherical inclusions. The inclusions of these types were extracted from all the ingots examined, and it was ascertained that these inclusions exist in every mechanical capped steel. As to the general proportion of occurrence among the three, the semicircular type accounts for about $50 \%$, while the remaining half is comprised of the plane and bowl types. The latter two were extracted only from low-carbon materials but not medium-carbon materials. The shape of inclusion, in most cases, depends on the heat given to the ingot. However, even when the temperature is identical, various shapes were observed in different ingots, in some rare cases.

These three types were commonly named the "tortoise shell-shape" inclusion (hereafter it will be so called as mentioned above unless specifically designated).

Further, sandlike alumina was found, but only in

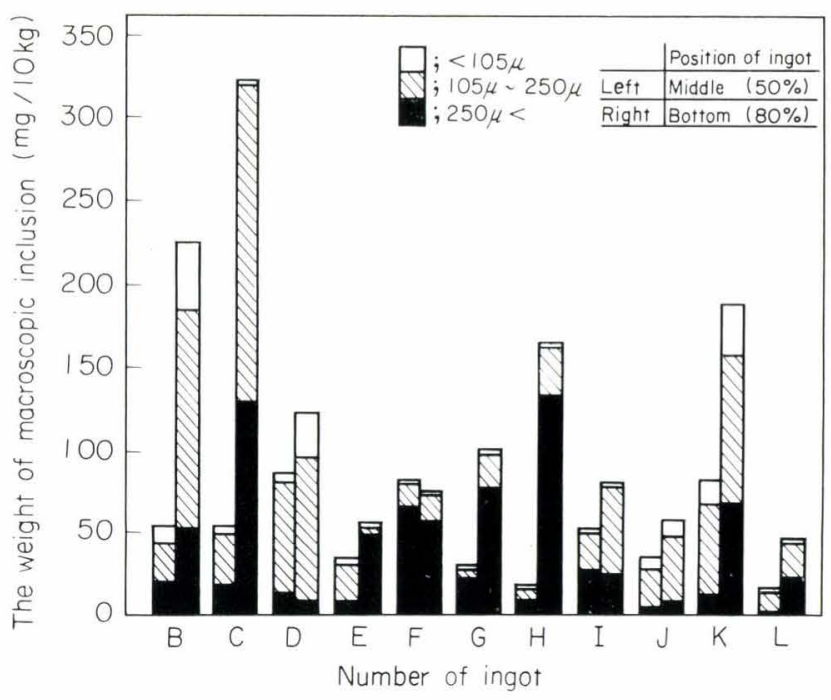

Fig. 1. Weight of macroscopic inclusion with different size extracted from the rim zone 
a small quantity. However, some of them were as large as $1000 \mu$ in the diameter. It is a squarish crystalline substance, and mostly translucent; this kind of inclusion is termed the lumpy alumina.

Up to the present, there is only the work done by Asano, et al. ${ }^{2)}$ on the macroscopic inclusions in the rim zone by using open-top steel samples as far as the authors know. Other papers by Kajioka ${ }^{5)}$ on opentop steel, and by Hisayoshi, et al. ${ }^{6)}$ and Katayama, et $a l .{ }^{7)}$ on capped steel cover neither tortoise shell-shape inclusions, nor large, single-substance alumina.

\section{Sticking State of Inclusions}

The sticking state of these inclusions in the rim zone is shown in the upper row of Photo 3.

The tortoise shell-shape inclusions, first of all, are built up in the interior surface of blow hole, as seen in the photograph, and are sticking at random in the peripheral direction with no specific behavior. On the other hand, they are distributed with a certain orderliness in the depthwise direction from the front (surface side of ingot) of lenticular blow hole to the rear (core side), and are sticking denser toward the rear end. A microscopic photograph of this inclusion is given in the lower row, where the white area designated the matrix and the black region corresponds to the blow hole. Thus it was made clear that the inclusion is captured and built up on the interior surface of blow hole, being in close contact with the matrix.

The lumpy alumina, secondly, as assumed from the photograph, is captured just as popped out onto the inner surface of blow hole and is easily movable with a single push. A large number of lumpy aluminas is captured by the blow holes existing in the core, rather than by the lenticular blow holes (into A) - this phenomenon will be discussed later.

Up to the present, there is no available literature referring the macroscopic inclusions captured in the lenticular blow holes. Only Hisayoshi, et al. ${ }^{8)}$ identified the existance of $\mathrm{FeO}-\mathrm{MnO}$ inclusions having the size of few microns by observing the interior surface of lenticular blow holes in open-top steel with on X-ray microanalyzer and pointed out that it occurs more

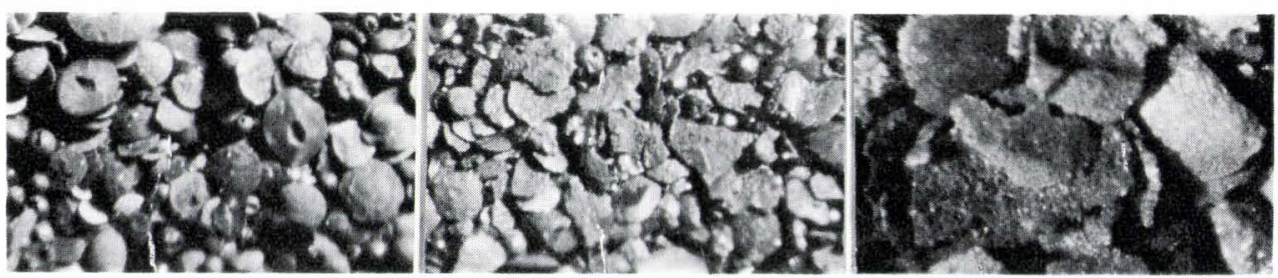

Semi circular type $(\times 32)$

Plane type $(\times 32)$

Bowl type $(\times 16)$

Photo. 2. The tortoise shell-shape inclusion extracted from the rim zone
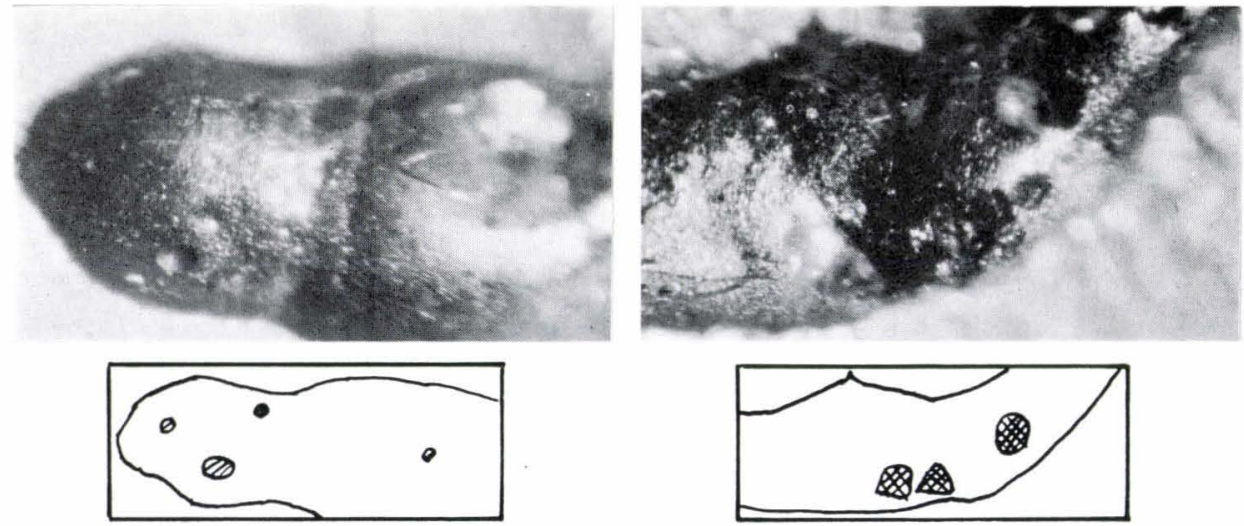

Tortoise shell-shape inclusion $(\times 50)$

Lumpy alumina $(\times 50)$
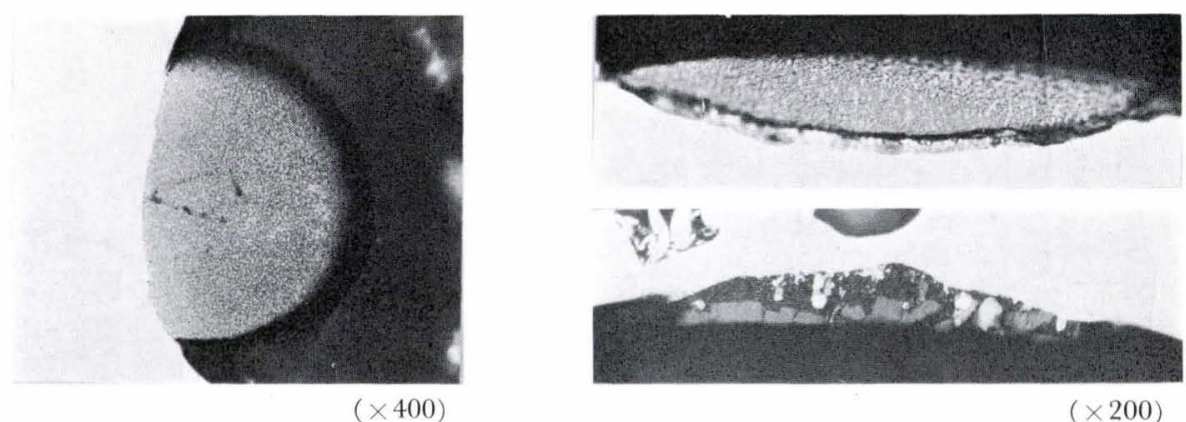

Upper: A macroscopic inclusion captured by lenticular blow hole

Lower: A tortoise shell-shape inclusion

Photo. 3. The sticking state of macroscopic inclusions and the microphotograph of tortoise shell-shape inclusions 
frequently in the low-sulfur materials.

4. Distribution of Macroscopic Inclusions in the Rim Zone

Generally speaking, the distribution of macroscopic inclusions in the rim zone in the heightwise direction of ingot becomes denser, as it approaches to the bottom of ingot as clearly shown in Figs. 1 and 6 . It is natural from the behavior of occurrence that tortoise shell-shape inclusions and lumpy alumina do not exist without blow holes. In other words, they are not observed in the region where lenticular blow holes are not formed.

Figure 2 shows the distribution of such inclusions in the thicknesswise direction of the rim zone which is observed at a $20 \%$ level above the ingot bottom.

In this examination, as shown in the photographs of microetched corner samples given on the top of the graph, the rim zone ( 80 to $100 \mathrm{~mm}$ ) was divided into four regions and the sample was then subjected to the slime test the dimensions, weight, and mean diameter of blow holes.

Further, upon the completion of electrolysis, the weight and the dimensions of remaining samples were measured, and the volume of blow holes in each sample was deducted from the values obtained.

To begin with the distribution of blow holes in the rim zone, only few needlelike blow holes are identified around the solid skin, whereas they grow in number at the region deeper than 20 or $25 \mathrm{~mm}$ and the density becomes roughly constant in this region. The amount was about 10 to $20 \%$ in the volumetric ratio, though it depends on the ingot.

The mean diameter of lenticular blow holes be- comes larger in deeper regions. Elucidating it from the relation between the mean diameter and the quantity of blow holes, the mean diameter of blow holes becomes larger and its number lessens as it goes into deeper region.

Secondly, with respect to the distribution of macroscopic inclusion, there is a marked trend that the tortoise shell-shape inclusions are growing rapidly in number at it becomes deeper. Their distribution and the mean diameter of lenticular blow holes establish a remarkable correspondence. This relation is graphically represented in Fig. 3 .

This characteristic curve means that the number of inclusions captured by blow holes increases as the diameter of blow hole becomes large, i.e., the rimming weakens. Here arises a complete contradiction to the report by Asano, et al. $\left.{ }^{2}\right)$ who measured a quantity of macroscopic inclusions in the superficial zone.

Considering that they did not mention the presence of tortoise shell-shape inclusions, this disagreement may be due to the fact that spherical inclusions might be found more plentifully in the superficial zone of open-top steel.

On the other hand, no orderliness was found with regard to the distribution of lumpy alumina or spherical inclusions, due mainly to its scare occurrence.

Meanwhile, the fact shown in Fig. 2 that the blow hole volume and mean diameter of ingot $\mathrm{G}$ are largely different from those of ingot $\mathrm{F}$ or $\mathrm{H}$ may be derived from a drastic difference in the rimming pattern as is clear from the microphotographs in the upper rowthe rimming pattern of ingot $\mathrm{F}$ or $\mathrm{H}$ is active in the initial stage but becomes dull in the later stages while

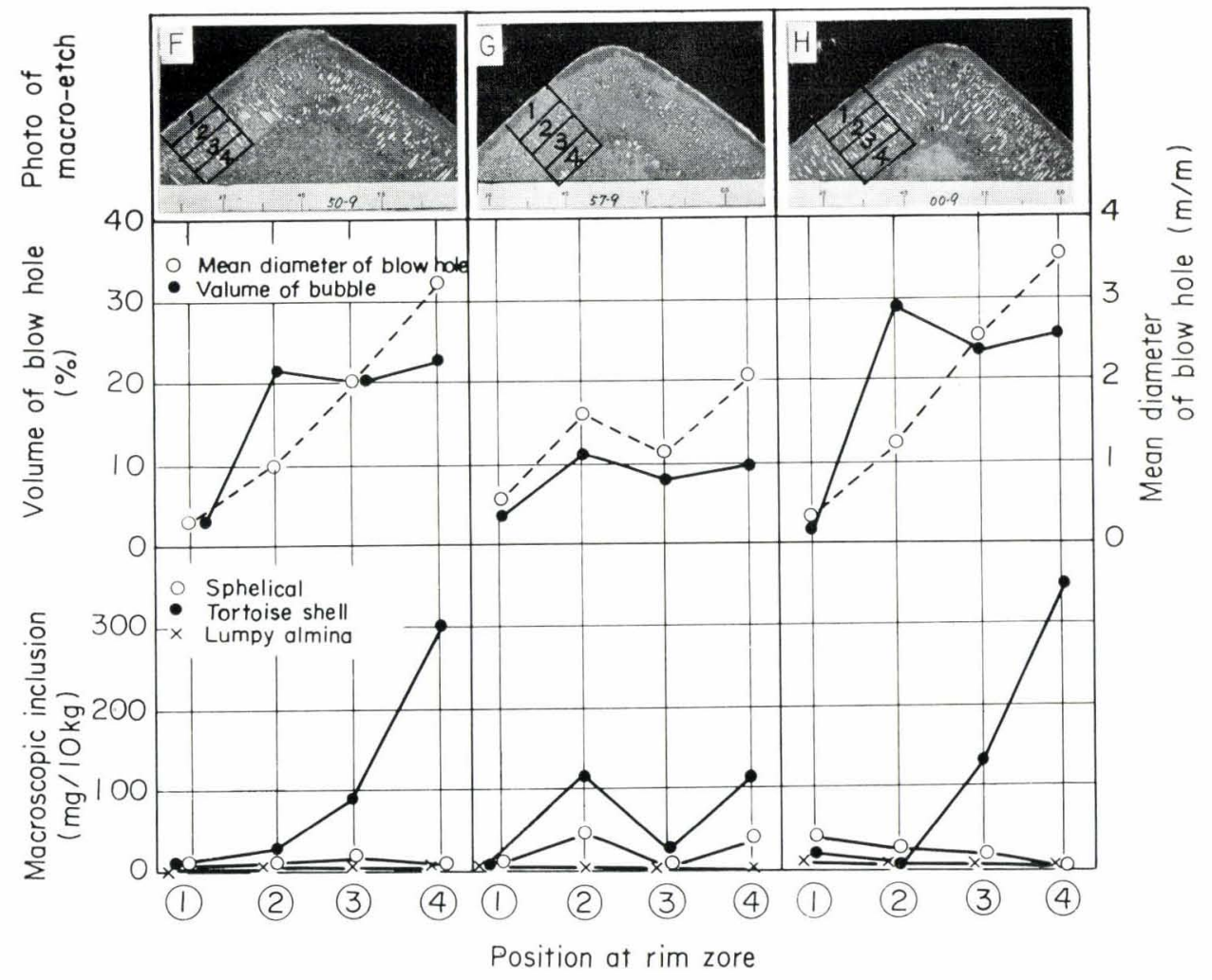

Fig. 2. The distributions of volume and diameter of blow hole, and of inclusion in the rim zone (at the level of $20 \%$ height from the bottom) 


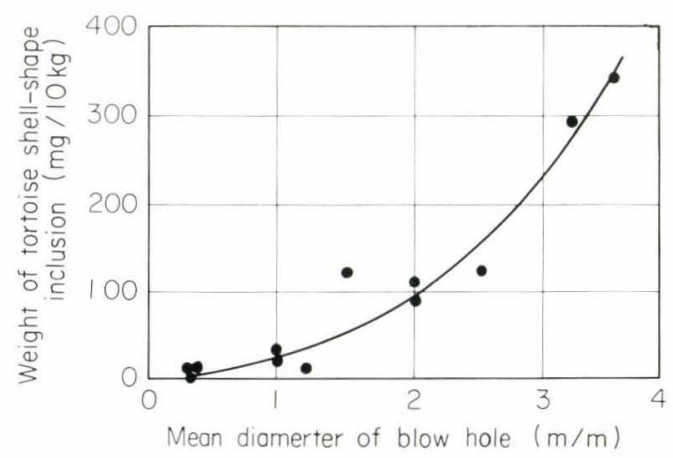

Fig. 3. Relationship between tortoise shell-shape inclusion and mean diameter of blow hole

that of ingot $\mathrm{G}$ is first dull, active in the middle stage, and then becomes dull again, perhaps on account of higher temperature of molten steel.

\section{Distribution of Microscopic Inclusions in the Rim} Zone

The cleanliness of inclusions in the rim zone and their mean diameter observed in the measuring views (60 views) were measured in $10 \mathrm{~mm}$ pitch increment along the depthwise direction, and the results obtained are shown in Fig. 4.

The measuring level was $20 \%$ above the bottom, and the intended measuring was limited only to the inclusions in the matrix.

Therefore, it is known that the cleanliness is not so variable in the superficial $50 \mathrm{~mm}$ deep zone, whereas the inclusions increase rapidly as it approaches to the rim-core border, and the cleanliness becomes worst at the outmost region of core wherein the rimming is terminated.

Since the rimming is originally feeble and the weakening of boiling phenomenon becomes slower along with the advancement of solidification, the cleanliness of medium-carbon rimmed steel does not deteriorate so badly as in the case of low-carbon rimmed steel.

Speaking of the mean diameter of inclusions, it is very small in the case of low-carbon steel, namely 3 to $5 \mu$ at the superficial region and 8 to $9 \mu$ near the rim-core border. In the case of medium-carbon high-manganese rimmed steel, it is as large as 10 to $12 \mu$ at the rim-core border, and slightly large inclusions are remaining. The maximum particle diameter of inclusions observed in all the views at each measuring point, was 10 to $30 \mu$ at the superficial zone, and 20 to $70 \mu$ around the rim-core border.

In this way it was discovered that the inclusions existing in the matrix are generally very small, and that most of large inclusions are captured by blow holes.

\section{Composition of Macroscopic lnclusions}

Photograph 4 shows the typical results obtained by EPMA analyses of macroscopic inclusions extracted from the rim zone.

The left four columns are tortoise shell-shape inclusions, where large galaxites are detected, and $\mathrm{MnO}$, Mn-wüstite, noncrystalline $\mathrm{MnO}-\mathrm{SiO}_{2}$, and $(\mathrm{Mn}-\mathrm{Fe}) \mathrm{S}$ are seen. The right two columns show the

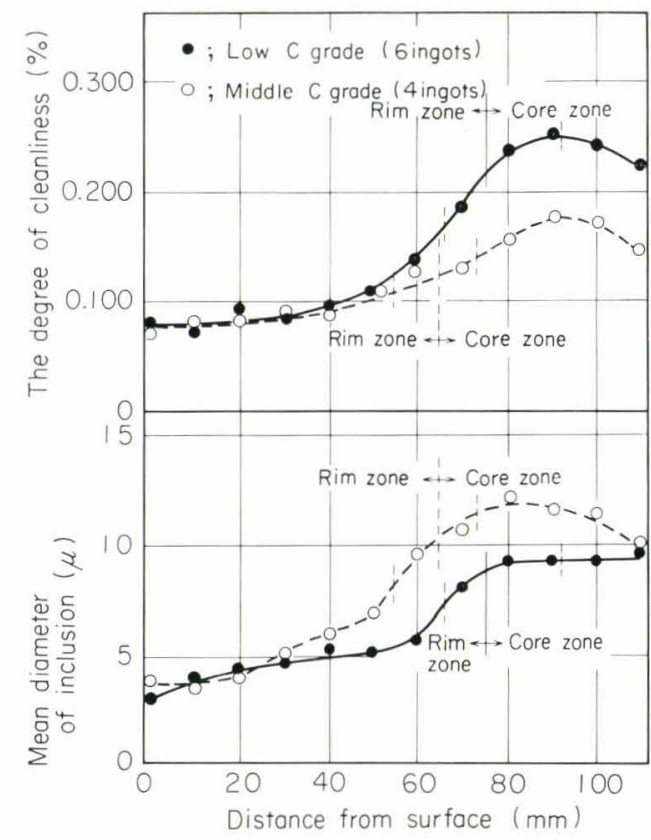

Fig. 4. The degree of cleanliness and the mean diameter of inclusions in the rim zone

analysis of lumpy alumina composed of $\mathrm{Al}_{2} \mathrm{O}_{3}$ with only a slight amount of $\mathrm{SiO}_{2}$ and $\mathrm{CaO}$ scattered partially. The average compositions of tortoise shell and spherical inclusions were further determined by chemical analysis separately.

Chemical compositions of typical tortoise shellshape inclusions found in low-carbon rimmed steel are 17 to $37 \% \mathrm{FeO}, 34$ to $41 \% \mathrm{MnO}, 17$ to $30 \% \mathrm{Al}_{2} \mathrm{O}_{3}$, 6 to $11 \% \mathrm{SiO}_{2}$, and 1 to $10 \% \mathrm{CaO}$, whereas the spherical inclusions, contain 22 to $23 \% \mathrm{FeO}, 37 \% \mathrm{MnO}, 10$ to $23 \% \mathrm{Al}_{2} \mathrm{O}_{3}$, and 17 to $19 \% \mathrm{SiO}_{2}$ - though it was the analytical results of two samples because the inclusions extracted were very small in quantity.

In the case of medium-carbon, high-manganese rimmed steel, the chemical composition of the inclusion was as follows: 10 to $30 \% \mathrm{FeO}, 40$ to $52 \% \mathrm{MnO}$, 4 to $17 \% \mathrm{Al}_{2} \mathrm{O}_{3}, 11$ to $17 \% \mathrm{SiO}_{2}$, and 2 to $5 \% \mathrm{CaO}$. When they are plotted on the (Fe, Mn) $\mathrm{O}-\mathrm{SiO}_{2}-$ $\mathrm{Al}_{2} \mathrm{O}_{3}$ equilibrium diagram, ${ }^{9)}$ Fig. 5 is obtained.

In the case of low-carbon rimmed steel, both the tortoise shell and spherical inclusions are coexisting in the galaxite region, but only the spherical inclusions are found in the region of slightly higher $\mathrm{SiO}_{2}$

On the other hand, they are existing in a MnOrich region in the case of medium-carbon high-manganese rimmed steel.

\section{Macroscopic Inclusions in the Core}

\section{Results of Macroscopic Inclusion Extraction}

Figure 6 indicates the results of extraction of macroscopic inclusions from various spots in the axial center of ingot $\mathrm{A}$.

In comparison with the conventional findings ${ }^{2}$ regarding the macroscopic inclusions in the core of capped steel, there is no difference in the distribution and the quantity as far as the examination from TM zone $(25 \%)$ to the bottom $(100 \%)$ is concerned.

On the contrary, peculiarities were admitted in the 


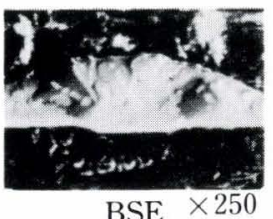

BSE $\times 250$

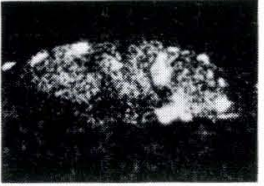

$\mathrm{Fe}$

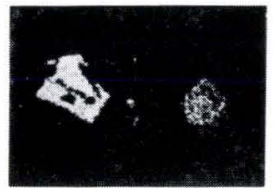

$\mathrm{Al}$

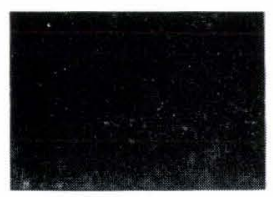

$\mathrm{Ca}$

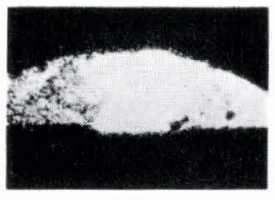

Mn

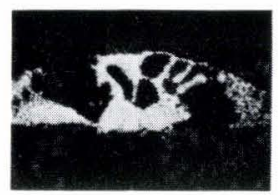

$\mathrm{Si}$

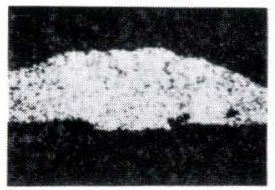

$\mathrm{O}$

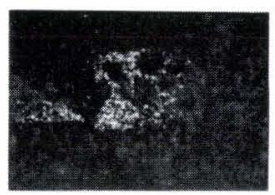

$\mathrm{S}$

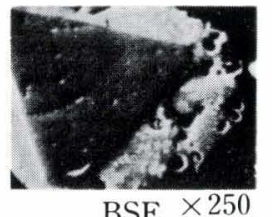

$\mathrm{BSE} \times 250$

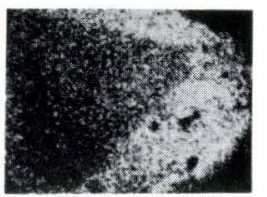

$\mathrm{Mn}$

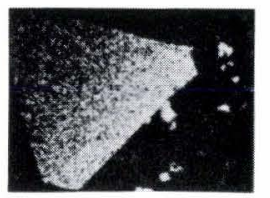

$\mathrm{Al}$

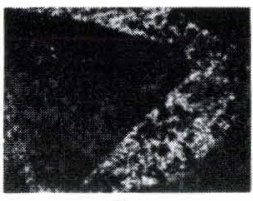

$\mathrm{Ca}$

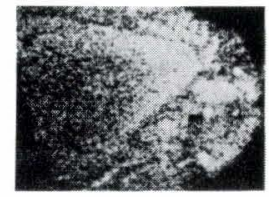

$\mathrm{Fe}$

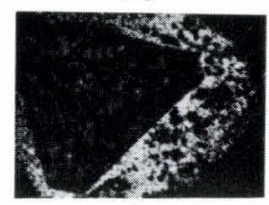

$\mathrm{Si}$

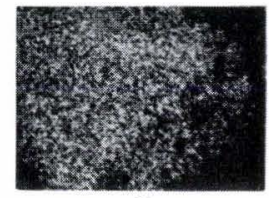

O

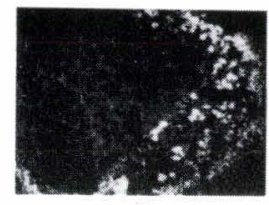

$\mathrm{S}$
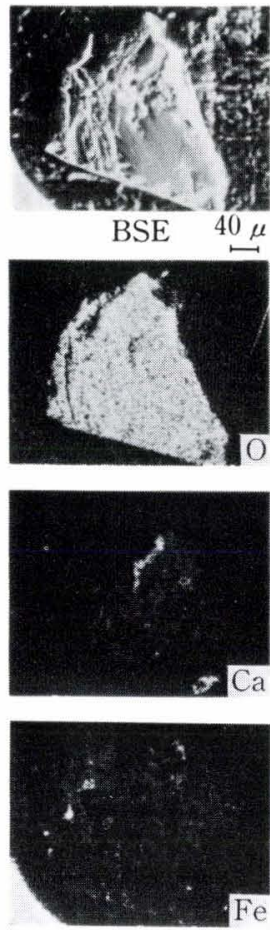
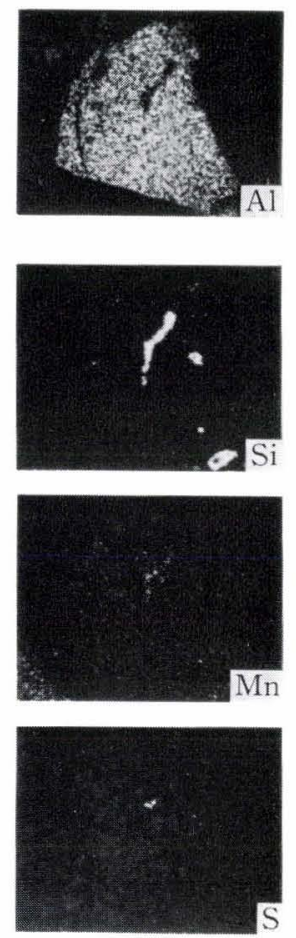

Tortoise shell-shape inclusion lumpy alumina

Photo. 4. Analytical results of tortoise shell-shape inclu sion and lumpy alumina obtained by EPMA

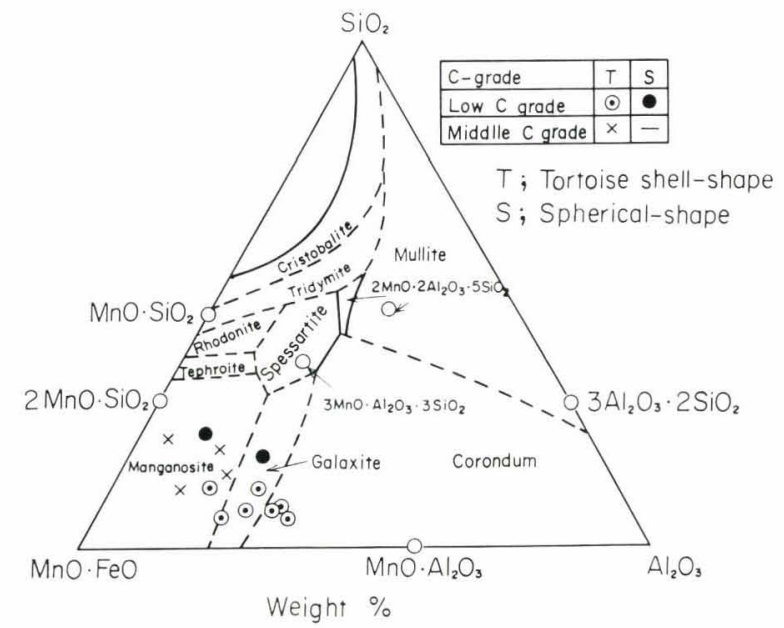

Fig. 5. Composition and oxide phase of macroscopic inclusion extracted from the rim zone

top to $20 \%$ deep region and the shoulder part, and a large quantity of macroscopic inclusions were extracted therefrom.

Most of these inclusions were very large in size and some superlarge ones measured $250 \mu$ or even $1000 \mu$ in size. Such extraction of macroscopic inclusion in quantities from the top area has not been reported up to the present.

These supermacroscopic inclusions extracted were mostly lumpy aluminas as explained later.

It is characteristic that the lumpy aluminas in the core were outstandingly numerous in the top region, and that they were also extracted at other position, though the quantity was somewhat variable except the massy zone at the bottom and the concentrated segregation area (TM zone). A typical example is

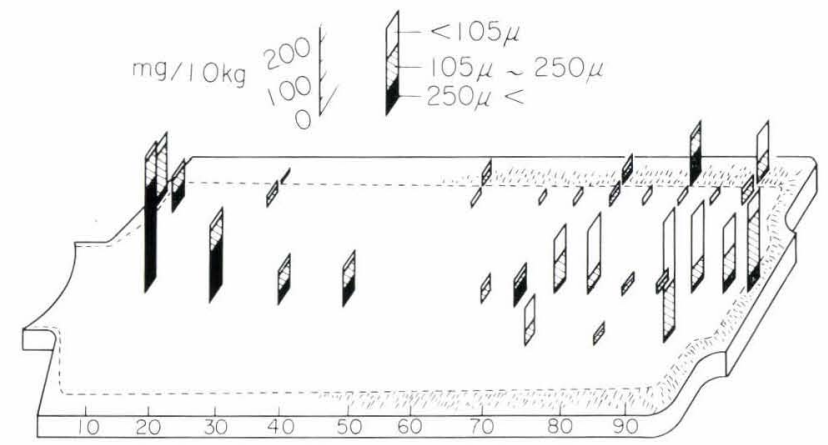

Fig. 6. The distribution of macroscopic inclusions in the ingot

shown in Photo. 5. This inclusion is extracted from the position $240 \mathrm{~mm}$ deep from the ingot surface (corresponding to $1 / 4$ of the width) in the $25 \%$ level from the bottom; squarish, sandlike particles shown in this photograph are lumpy aluminas and most of them are apparently large.

\section{Sticking State of Lumpy Alumina}

From the investigations made to find how, or in what from such macroscopic lumpy aluminas are captured in the core, it is found that all of them are captured by the blow holes in the core.

Just like the inclusions captured by lenticular blow holes in the rim zone (upper pictures in Photo. 3), they were identified to be captured as being popped out into the blow holes.

As to the tortoise shell-shape inclusion, however, only a few particles were discovered in the core, this fact indicates that the tortoise shell-shape inclusions captured by blow holes in the core are very small in number. 
3. Compositions of Inclusions Found in Various Locations

The inclusions extracted from various positions of ingot were classified by the size as shown in Fig. 7, taking the solidification into consideration, and the average composition of the inclusions classified by the chemical analysis. The findings illustrated the features of each position as follows: nearly $90 \%$ of the particles larger than $250 \mu$ obtained from the axial center of top part (1) was $\mathrm{Al}_{2} \mathrm{O}_{3}$, and about $70 \%$ of those existing in the core side of shoulder part (9) was also $\mathrm{Al}_{2} \mathrm{O}_{3}$. Thus, it is clear that the majority of macroscopic inclusions in the core of top part is lumpy alumina.

As it is understood from the fact that the sulfur content of macroscopic inclusions in the concentrated segregation zone is about $18 \%$, flakelike $\mathrm{MnS}$ and

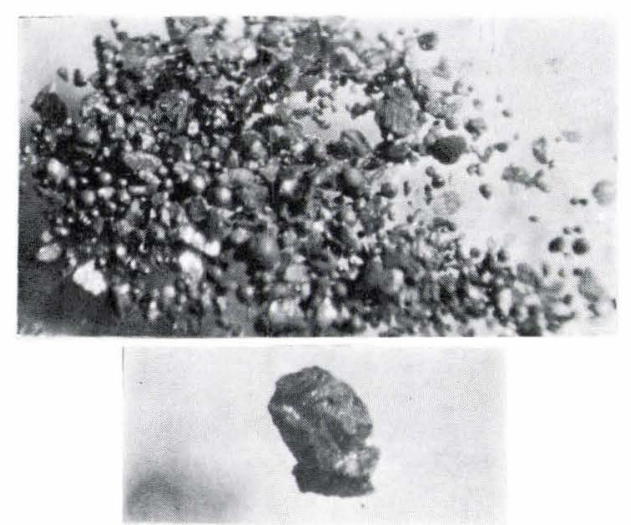

Upper: Macroscopic inclusion in the core zone (at the level of middle-bottom and the depth of $240 \mathrm{~mm}$ from the surface $)(\times 32)$

Lower: Lumpy alumina $(\times 10)$

Photo. 5. The extracted inclusion
$\mathrm{FeS}$, highest in the content, were extracted as much as $50 \%$. Both of them are very fragile and have golden color. The rest of the inclusion was mostly $\mathrm{Mn}$ wüstite.

For the inclusions smaller than $250 \mu$, Mn-wüstite grows in number and becomes predominant in the class of $100 \mu$ or less.

With respect to the inclusions in the bottom massy zone (4) and (5), there is a characteristic trend, rich in $\mathrm{SiO}_{2}$ and poor $\mathrm{Al}_{2} \mathrm{O}_{3}$, for large size inclusions. If it is considered from the relation to the tortoise shellshape inclusion in the rim zone, it is deduced that they are generated and are captured in the same period, because the tortoise shell-shape inclusions are close to the average values of macroscopic and microscopic inclusion in the massy zone.

Particularly, from the fact that the content of $\mathrm{CaO}$ in the inclusions, possibly derived from converter slag, and the content of $\mathrm{SiO}_{2}$ which is chiefly attributable to converter slag or refractories are very high, these inclusions seem to be formed by the combination of foreign inclusions ${ }^{1)}$ with $(\mathrm{Fe}, \mathrm{Mn}) \mathrm{O}$ generated by the air oxidation or produced at the front of solidification surface.

The results obtained were plotted in the (Fe, Mn) $\mathrm{O}-\mathrm{SiO}_{2}-\mathrm{Al}_{2} \mathrm{O}_{3}$ ternary diagram ${ }^{9)}$ and are graphically shown in Fig. 8. In this diagram, it is quite characteristic that there is a clear difference between top part inclusions and bottom part ones.

\section{Discussions}

\section{Tortoise Shell-shape Inclusions}

It was recognized that there were great quantities of large oxide inclusions in the rim zone, most of which were captured inside the lenticular blow holes.

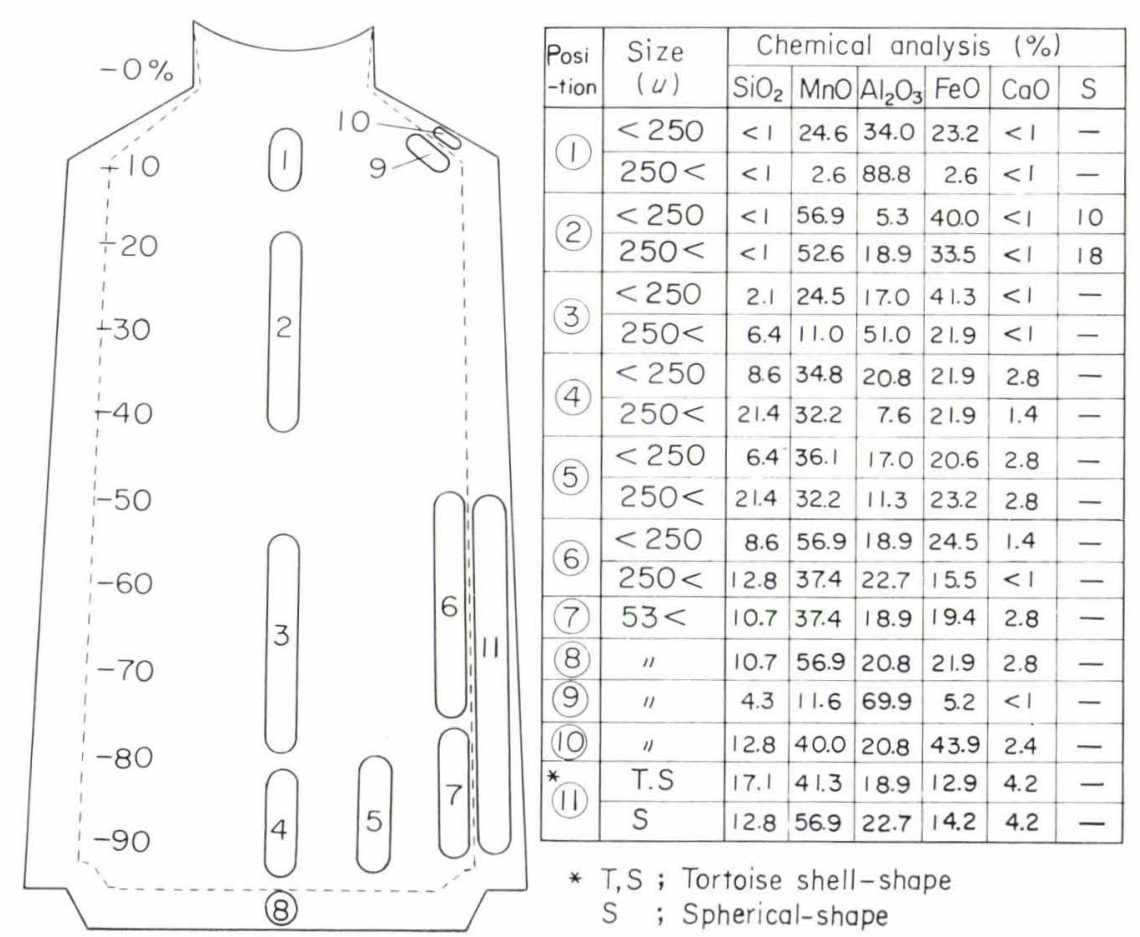

Position of sample
Fig. 7. Chemical compositions of macroscopic inclusions extracted from the respective zones of the ingot 


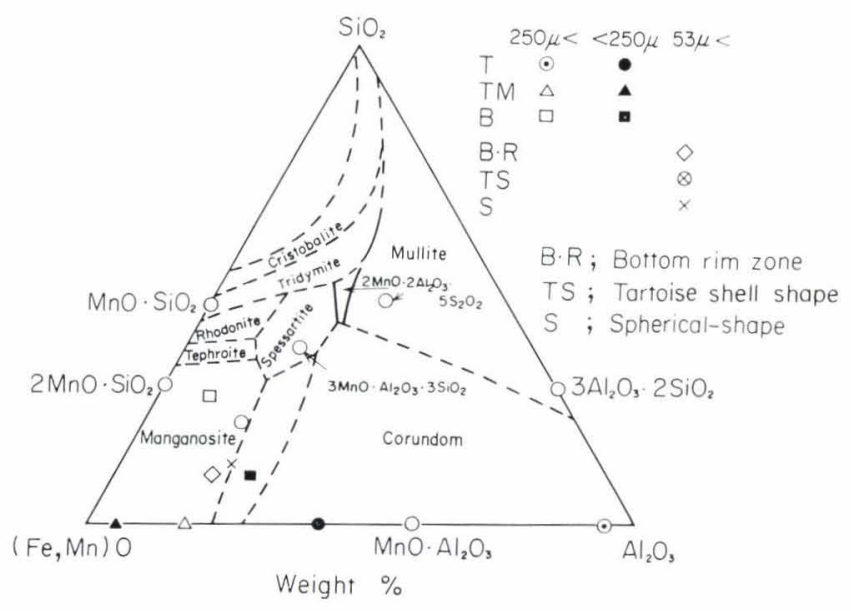

Fig. 8. Composition and oxide phase of macroscopic inclusions extracted from the core zone

As it is obvious from Fig. 3, such inclusions are relative to the volume and the diameter of blow hole. Since the blow hole may well be said to represent the rimming itself, the relation between blow hole and rimming will be discussed below.

Firstly, in its relation, a strong rimming means the generation of numerous $\mathrm{CO}$ blow holes from the solidification surface, or a strong cleaning power at the front of solidification surface. Hence, the number of remaining blow holes becomes large, but their diameters are small. This agrees with the result of Fig. 2.

If the inclusions captured by these blow holes are considered, it must be first noted that they are hardly captured due to the small surface area of blow hole.

Secondly, a strong cleaning power will make them hard to be captured because the front of solidification surface becomes smooth (remelting of dendrite).

Thirdly, the accession of inclusions to the solidification surface is prevented by the detached blow holes of large quantity. In other words, the inclusions are captured by the blow holes generated like as a curtain and float up together with blow holes, and thus the inclusions do not reach to the solidification surface.

To the contrary, if the rimming weakens, the cleaning power drops, blow holes are hardly detached from the solidification shell, and the diameter of blow hole increases. Consequently, the inclusions are more easily captured by remaining blow holes due to the increasing surface area, and the detaching and floating up of blow holes are retarded at the same time, allowing more and more inclusions to remain inside the blow holes.

Since the floating and separation of macroscopic inclusions in the molten steel progresses, the number of inclusions is estimated to be greatly reduced right after casting. But, in spite of such a trend, the number of tortoise shell-shape inclusions increases because the uncaptured amount due to the weakened rimming is much larger than such an increment.

This explanation is only a qualitative deduction, but it will be enough to illustrate the behavior from the phenomenal standpoint.

\section{Distribution of Macroscopic Spherical Inclusions}

The number of tortoise shell-shape inclusions increases in the deeper zones, and microscopic inclusions, demonstrated in the inclusion cleanliness chart of Fig. 4, also increases in the areas closer to the rimcore border.

In the meantime, macroscopic spherical inclusions are almost constant over the whole area of ingot as shown in Fig. 2. This characteristic may be explained as follows.

The amount of inclusions captured by solidification shells is determined by the strength of rimming, that is, in terms of the cleaning power at the front of solidification surface and the amount of inclusions existing in molten steel.

Thereby, inclusions are abundant during casting or right after casting, but they are hard to be captured on account of the strong rimming action.

This is proved by the phenomenon in which, as to ingot $\mathrm{G}$ whose rimming was weak right after casting, spherical inclusions increased in number at point 2 which is corresponding to the period during casting through right after casting.

As the solidification further develops, the rimming weakens and, simultaneously, the number of macroscopic inclusions in molten steel will be decreased by the flotation and separation.

Whence it is considered that the amount of macroscopic spherical inclusions captured does not increase, too.

Meanwhile, as microscopic inclusions are hard to float and separate, the volume does not diminish even if the solidification advances; it is estimated, therefore, that the amount captured increases as the rimming weakens.

\section{Lumpy Aluminas in the Core}

In the capped steel, whether in the core or in the rim zone, an abundant extraction of lumpy aluminas was recognized, which may be summarized as follows:

(1) Lumpy aluminas are all captured in blow holes, whether in the core or in the rim zone.

(2) They are extracted most plentifully from the zone where blow holes are aggregated, in the core of top part.

(3) They are also extracted from the level close to the bottom (MB zone) and $240 \mathrm{~mm}$ deep from the ingot surface.

The following discussions will refer to these points.

Starting with the solidification, the time required for $240 \mathrm{~mm}$ deep solidification will be slightly longer than $90 \mathrm{~min}$ if $k$ factor $=25$. This period is the longest at the $10 \%$ axial center level of the top part.

Therefore, the problem is how single-substance aluminas having the size of 50 to $1000 \mu$ were existing in the molten steel for such a long period of time. It may be either floating in the molten steel of suspending by other mechanism.

The former assumption seems to be improbable if the size of alumina, the time elapsed, and the location in the steel ingot are taken into account. If they 
were floating, it is doubtful that they could remain as a pure $\mathrm{Al}_{2} \mathrm{O}_{3}$ single substance for such a long period of time without being affected by the molten oxides of $\mathrm{Fe}-\mathrm{Mn}-\mathrm{O}$ system.

This, again, opposes the equilibrium relation of $\mathrm{Al}_{2} \mathrm{O}_{3}-(\mathrm{Fe}, \mathrm{Mn}) \mathrm{O}$ system.

Therefore, the latter assumption seems to be more probable.

As one of the other mechanisms, the protective transfer by blow holes can be taken into consideration.

This explanation coincides with the fact that the lumpy aluminas were found only in the blow holes, or that they existed most plentifully in such a zone as the axial center of top part where blow holes are regarded to accumulate in a large quantity after floating up.

As shown in the microetching photograph of Fig. 9 , such blow holes in the core are lined up in an inverted $\mathrm{V}$ form from the rim-core border to the axial center; some of them are continuous though they are interrupted two-dimensionally.

These blow holes give a historical track of the progress of solidification, the shrinkage of volume, and the growth of $\mathrm{CO}$ blow holes to compensate the loss, in succession.

In other words, it is assumed that the blow holes, while growing up, are transporting lumpy aluminas. This behavior is expressed by a model form shown in Fig. 9.

In this way, large lumpy aluminas could exist in the $\mathrm{MB}$ zone, and, again, it may be possible to exist as $\mathrm{Al}_{2} \mathrm{O}_{3}$ single substances if they are protected inside the blow holes.

Here, a thick concentration of lumpy aluminas at the top part can be understood as a matter of course, by considering that the blow holes containing lumpy aluminas are partially separated in the process of solidification to float and accumulate at the top part.

However, several problems remain unsolved. One of them is the reason why the tortoise shell-shape inclusions are scarcely existing in the blow holes found

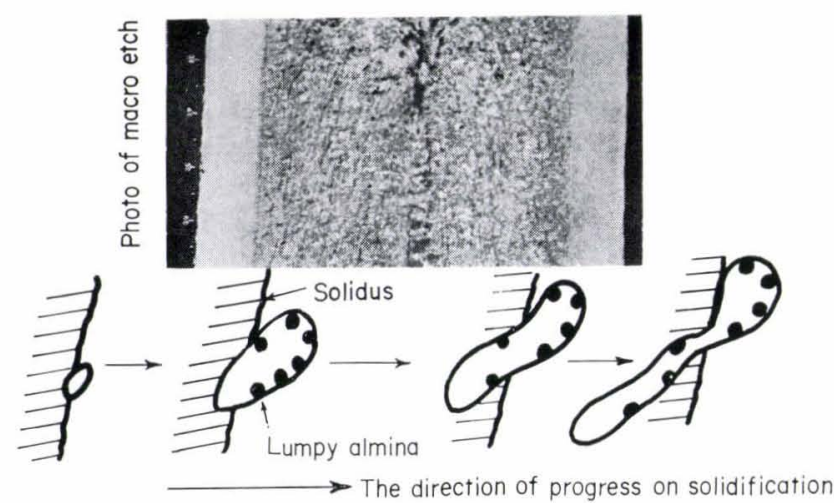

Fig. 9. The distribution of blow holes in the core zone and the model for the traveling lumpy alumina inclusion in the core. The second question is why the tortoise shell-shape inclusions are not transferred. The third problem is when lumpy aluminas are captured by blow holes, supposing the estimation above is all correct.

\section{Conclusion}

Several new facts were obtained from the investigations on the inclusions in the large-sized, mechanical capped steel. The results obtained are summarized as follows.

(1) Macroscopic inclusions are most abundantly found in the core of top side-most of which are lumpy aluminas.

(2) Lumpy aluminas are found in every possible blow holes existing in the core or in the rim zone.

(3) A large quantity of tortoise shell-shape inclusions of $\mathrm{Fe}-\mathrm{Mn}-\mathrm{Si}-\mathrm{Al}-\mathrm{O}$ system is existing in the rim zone, and they are all captured by lenticular blow holes. They are, however, rarely captured by blowholes in the core.

(4) The distribution of such tortoise shell-shape inclusions agrees almost perfectly with the distribution of lenticular blow holes and the blow hole mean diameter, and the amount of inclusions captured is greatly affected by the strength of rimming.

\section{Acknowledgements}

Last of all, the authors wish to express their sincere appreciation to Mr. Kurita, Assistant Manager of the Laboratory, and Mr. Nishizawa, Assistant Manager, for their invaluable assistance and advice through the entire course of this investigation. The authors are also much indebted to Mr. Hashio, Sub-chief, and his staff at No. 1 Steelmaking Plant for their cooperation in casting of test pieces and sampling. Sincere thanks are due also to Mr. Domen, Work Chief, Mr. Takeuchi, Plant Chief, and the staff members of Performance Control Group for high-precision testing of countless samples.

\section{REFERENCES}

1) K. Asano, T. Ōhasi and Y. Nuri: Tetsu-to-Hagané, 54 (1968), 643.

2) K. Asano, T. Saeki and Y. Nuri: Tetsu-to-Hagané, 57 (1971), 2010.

3) T. Ikeda, K. Sumitomo, K. Marukawa and S. Ura: Tetsuto-Hagané, 57 (1971), 2022.

4) K. Morinaga, A. Ōba and Y. Itoh: Tetsu-to-Hagané, 49 (1963), 1663.

5) H. Kazioka: Tetsu-to-Hagané, 54 (1968), 696.

6) M. Kuba, K. Tasaka, Y. Itoh and H. Maeda: Tetsu-toHagané, 58 (1972), S352.

7) H. Katayama and H. Kazioka: Tetsu-to-Hagané, 56 (1970), S410.

8) M. Kuba, K. Tasaka, Y. Itoh and H. Maede: Tetsu-toHagané, 58 (1972), 41.

9) E. F. Osborn and A. Muan: Electric Furnace Steelmaking, II, Interscience Publishers, New York (1963), 256. 